\title{
Cancer risk in primary biliary cirrhosis: a study in northern England
}

\author{
D Howel, J V Metcalf, J Gray, W L Newman, D E J Jones, O F W James
}

\begin{abstract}
Background-Suggestions that breast cancer may be more common in patients with primary biliary cirrhosis (PBC) have been challenged. It has recently been proposed that total cancer rates may be higher in patients with PBC, as well as liver cancers.
\end{abstract}

Aims-To investigate these proposals on a strictly defined case series.

Subjects-A total of 769 prevalent or incident PBC patients with "definite" or "probable" disease detected in a defined area of the north-east of England during 1987-94.

Methods-Cancer events and deaths were identified by obtaining information from one or more of the following sources: Office for National Statistics (ONS) Central Registers, Regional Cancer Registry, and clinical case records. Standardised cancer incidence (SIR) and mortality ratios (SMR) were calculated using the local region as the standard population.

Results-There were 97 cancer events during 1987-96. SIR from cancer registrations for all cancers was 1.7 (95\% confidence interval (CI) 1.3 to 2.2), for liver cancer was 74 (95\% CI 32 to 146), and for breast cancer was 1.1 (95\% CI 0.4 to 2.4$)$. SMR for all cancers was $1.8(95 \%$ CI 1.4 to $2.4)$, for liver cancer was $39(95 \%$ CI 20 to $68)$, and for breast cancer was 0.4 (95\% 0.1 to 1.6 . The results were similar after excluding the first year of follow up after PBC diagnosis.

Conclusions-There was some evidence of a small increase in overall cancer incidence and mortality in PBC patients. With the exception of liver cancer, it is unlikely that there is a high excess incidence for PBC patients from any cancer at a particular site, and specifically breast cancer.

(Gut 1999;45:756-760)

Keywords: primary biliary cirrhosis; cancer

Primary biliary cirrhosis (PBC) is a chronic autoimmune liver disease. While it is widely recognised that PBC may be associated with other autoimmune diseases, ${ }^{12}$ its possible relation with cancer is unclear. Early reports indicated increased risk of extrahepatic cancers in general, and breast cancer in particular, ${ }^{3-5}$ but evidence from later studies has been conflicting. Confirmation or refutation of the putative association between PBC and one or more malignancies is not merely of academic inter- est: it would affect patient management, in terms of extra surveillance. Possible explanations for the conflicting conclusions so far are: the variation in case finding methods; the small size of some case series; and the relatively short follow up time. We report the results of investigating the link between cancer and PBC in a large case series in north eastern England, in which multiple methods of case finding and strict diagnostic criteria have been used.

\section{Methods}

We have completed a comprehensive case finding study to determine the epidemiology of PBC. The methods used in this study have been described previously in more detail, ${ }^{6}$ but are described briefly below.

The study area comprised the adjacent health districts of Newcastle and North Tyneside, Gateshead and South Tyneside, Northumberland, Sunderland, and Durham in north eastern England: the total population is approximately 2 million.

This study included both definite and probable cases of PBC. The diagnosis of PBC was considered definite if all three of the following diagnostic criteria were met: antimitochondrial antibodies present in at least $1 / 40$; abnormal liver function tests; and diagnostic or compatible liver histology. Probable PBC was defined as any two of the above. Many cases in this cohort were eventually diagnosed as definite PBC, but as the final test (particularly the confirmatory liver biopsy) was often not done until some time after other evidence first occurred, the "date of presumed diagnosis" was taken to be the first date on which at least two of the above three investigations were found abnormal on reviews of clinical case records. This reduced any bias in diagnosis dates caused by intensive case finding.

Case finding was carried out for the period 1 January 1987 to 31 December 1994. The methods of case finding were: requests to all consultant gastroenterologists in the region for details of patients with PBC; hospital admission data on patients with ICD9 Code 576.1 or ICD10 Code K74-3 from all hospitals in the region; examination of autoantibody screen results from immunology laboratories in the region; and death certificates from all individuals dying in the region with PBC mentioned on the death certificate. All prevalent cases on 1 January 1987 and all cases diagnosed between 1987 and 1994 were included in the cohort.

Abbreviations used in this paper: HCC, hepatocellular cancer; PBC, primary biliary cirrhosis; SIR, standardised incidence ratio; SMR, standardised mortality ratio. 
Information on any cancer registration or death for patients in this cohort was obtained from the Office for National Statistics Central Registers and the regional cancer registry. As many cancers are not fatal, cancer incidence is of more interest than cancer mortality. However, given problems with completeness of cancer registrations, and the inability to obtain information at a national level for registrations beyond 1993, we have also used cancer mortality as a proxy. The suspected lack of completeness led us to also search patients' hospital notes for information about further cancer diagnoses, in order to see whether there was evidence of under registration. Information on cancer diagnosis from hospital notes was not used in the main analyses, as we were comparing the rates of registered cancer cases in PBC patients to those registered in the general population.

We excluded patients whose diagnosis of cancer preceded the diagnosis of PBC (prevalent cancers) from the main analyses. Although a general link between cancer and PBC would be of interest, the way this cohort was assembled was only suitable for investigating cancers occurring after PBC diagnosis: it is the diagnosis of $\mathrm{PBC}$ which makes subjects eligible for the cohort, and it is the date on which PBC was diagnosed from which follow up starts. The analyses have been carried out with and without the period of follow up for a year after PBC diagnosis. This was to reduce the impact of selection bias (the investigation of symptoms shortly after PBC diagnosis might increase the chance of cancer being investigated and diagnosed) and has been used in similar studies. ${ }^{7-9}$

Person years at risk were calculated for each patient starting immediately (and one year) after diagnosis of PBC, and ending with cancer registration, death, or end of follow up period. Annual cancer incidence and mortality rates by sex and five year age bands were obtained from the Northern and Yorkshire Cancer Registry for the northern region of England which covers the study area. Mortality rates were available up to 1996, but cancer registration data were only available up to 1993 , so follow up is to the end of 1996 for mortality calculations and the end of 1993 for incidence calculations. The standardised incidence and mortality ratios and their $95 \%$ confidence intervals based on the Poisson distribution were then calculated using the Northern region as the

Table 1 Characteristics of study cohort

\begin{tabular}{lcl}
\hline Characteristic & All & $\begin{array}{l}\text { Cancer diagnosis } \\
\text { post-PBC diagnosis }\end{array}$ \\
\hline No of patients with PBC & 769 \\
PBC prevalent at $1 / 1 / 87$ & $301(39 \%)$ & \\
PBC diagnosed between $1 / 1 / 87$ and 31/12/93 & $402(52 \%)$ & \\
PBC diagnosed between 1/1/93 and 31/12/94 & $66(9 \%)$ & \\
Female & $693(90 \%)$ & \\
Male & $76(10 \%)$ & 58 \\
No of cancer events 1987-96 & 65 & 16 \\
Cancer deaths & 23 & 8 \\
Non-fatal registered cancers & 9 & \\
Non-fatal cancers in hospital notes only & 239 & \\
Non-cancer deaths 1987-96 & & \\
\hline
\end{tabular}

PBC, primary biliary cirrhosis. standard population. As cancer at many sites is very rare, some sites were grouped together for analysis: these groupings were chosen prior to seeing any results.

\section{Results}

In total, 769 subjects fulfilled at least two case criteria for PBC within the study area and period. Table 1 outlines some characteristics of this cohort of patients. Thirty nine per cent of them had been diagnosed with PBC between 1969 and 1986 and were still alive at the start of the study period, and $61 \%$ were diagnosed within the case finding period $(9 \%$ after the end of 1993, so they could only contribute to the cancer mortality comparisons). The patients were predominantly female, with a median age at diagnosis of 63 years (range 17-91). In 97 individuals a cancer event occurred (death, registration, or information in hospital notes) during 1987-96, but in 15 of them the cancer event preceded diagnosis of PBC; these events were therefore excluded from later analysis. In 14 subjects the cancer registrations occurred before the diagnosis of PBC: the cancers had been registered between 17 days and 17 years before PBC was diagnosed. There were two stomach cancers, one respiratory, two breast, four reproductive, one urinary, and two haematological cancers. A table is available from the authors listing the cancer events which occurred after PBC diagnosis during the follow up period. This illustrates the distribution of cancer over the different sites, and over time, and the timing of PBC diagnosis, cancer registration, death from cancer, and first mention of cancer in hospital notes.

The total number of person years at risk of follow up in the incidence calculation was 2641 when using all incident cancers, and 2279 when omitting the first year of follow up after PBC diagnosis. Table 2 gives the standardised incidence ratios (SIR). These results show that for PBC patients there was a significantly raised risk of all cancers (SIR $=1.7)$, and considerably higher liver cancer risk $($ SIR $=74.3)$. However, the increased risk of cancer at all sites was no longer significant when the first year of follow up was excluded. There was no significantly increased risk of breast cancer with the SIR being 1.1 for total follow up period, and 1.0 excluding the first year after PBC diagnosis.

The total number of person years of follow up in the mortality calculation was 4190 years when including all deaths, and 3743 years when excluding cancer deaths within a year of diagnosis of PBC. The length of follow up for a subject varied between one day and 10 years with a median of 5.4 years. Table 3 gives the standardised mortality ratios (SMR). These results show that for PBC patients there were significantly raised risks of dying from all cancers $(\mathrm{SMR}=1.8)$, respiratory cancer $(\mathrm{SMR}=$ $2.4)$, and haematological cancers (SMR = 3.9); the risk of liver cancer was considerably raised $(S M R=38.6)$. The results were similar when the first year of follow up was excluded. The SMR for breast cancer was 0.4 and 0.3 for 
Table 2 Standardised incidence ratio (SIR) of cancer in patients with primary biliary cirrhosis (PBC)

\begin{tabular}{|c|c|c|c|c|c|c|}
\hline \multirow[b]{2}{*}{ Site of cancer (ICD9 code) } & \multicolumn{3}{|c|}{ Observed } & \multirow[b]{2}{*}{ Expected } & \multirow[b]{2}{*}{$S I R$} & \multirow[b]{2}{*}{$95 \% C I$} \\
\hline & All & Male & Female & & & \\
\hline \multicolumn{7}{|l|}{ Including all years of follow up } \\
\hline All cancers $(140-208)$ & 50 & 9 & 41 & 29.7 & 1.7 & 1.3 to 2.2 \\
\hline Digestive $(150-154,157)$ & 6 & 2 & 4 & 5.8 & 1.0 & 0.4 to 2.2 \\
\hline Liver (155) & 8 & 3 & 5 & 0.1 & 74.3 & 32.1 to 146 \\
\hline Respiratory (162) & 10 & 2 & 8 & 5.2 & 1.9 & 0.9 to 3.6 \\
\hline Breast (174) & 6 & - & 6 & 5.5 & 1.1 & 0.4 to 2.4 \\
\hline Reproductive (179-180, 182-183) & 4 & - & 4 & 2.7 & 1.5 & 0.4 to 3.8 \\
\hline Urinary $(188-189)$ & 1 & 0 & 1 & 0.4 & 2.9 & 0.1 to 16.2 \\
\hline Haematological (200-208) & 4 & 1 & 3 & 1.5 & 2.6 & 0.7 to 6.7 \\
\hline \multicolumn{7}{|c|}{ Excluding experience in first year after $P B C$ diagnosis } \\
\hline All cancers (140-208) & 36 & 6 & 30 & 26.5 & 1.4 & 0.9 to 1.9 \\
\hline Digestive $(150-154,157)$ & 3 & 1 & 2 & 5.2 & 0.6 & 0.1 to 1.7 \\
\hline Liver (155) & 7 & 3 & 4 & 0.1 & 72.7 & 29.2 to 150 \\
\hline Respiratory (162) & 7 & 0 & 7 & 4.6 & 1.5 & 0.6 to 3.1 \\
\hline Breast (174) & 5 & - & 5 & 4.9 & 1.0 & 0.3 to 2.4 \\
\hline Reproductive (179-180, 182-183) & 3 & - & 3 & 2.4 & 1.3 & 0.3 to 3.7 \\
\hline Urinary $(188-189)$ & 1 & 0 & 1 & 0.3 & 3.2 & 0.1 to 17.9 \\
\hline Haematological (200-208) & 2 & 1 & 1 & 1.4 & 1.5 & 0.2 to 5.3 \\
\hline
\end{tabular}

CI, confidence interval.

Table 3 Standardised mortality ratio (SMR) of cancer in patients with primary biliary cirrhosis (PBC)

\begin{tabular}{|c|c|c|c|c|c|c|}
\hline \multirow[b]{2}{*}{ Site of cancer (ICD9 code) } & \multicolumn{3}{|c|}{ Observed } & \multirow[b]{2}{*}{ Expected } & \multirow[b]{2}{*}{$S M R$} & \multirow[b]{2}{*}{$95 \% C I$} \\
\hline & All & Male & Female & & & \\
\hline \multicolumn{7}{|l|}{ Including all years of follow up } \\
\hline All cancers $(140-208)$ & 58 & 11 & 47 & 31.6 & 1.8 & 1.4 to 2.4 \\
\hline Digestive $(150-154,157)$ & 7 & 1 & 6 & 7.4 & 1.0 & 0.4 to 2.0 \\
\hline Liver (155) & 12 & 3 & 9 & 0.3 & 38.6 & 20.0 to 67.5 \\
\hline Respiratory (162) & 19 & 4 & 15 & 8.0 & 2.4 & 1.4 to 3.7 \\
\hline Breast (174) & 2 & - & 2 & 4.5 & 0.4 & 0.1 to 1.6 \\
\hline Reproductive $(179-180,182-183)$ & 3 & - & 3 & 2.6 & 1.2 & 0.2 to 3.4 \\
\hline Urinary $(188-189)$ & 2 & 0 & 2 & 0.3 & 6.6 & 0.8 to 23.6 \\
\hline Haematological (200-208) & 7 & 1 & 6 & 1.8 & 3.9 & 1.6 to 8.1 \\
\hline \multicolumn{7}{|c|}{ Excluding experience in first year after PBC diagnosis } \\
\hline All cancers $(140-208)$ & 46 & 9 & 37 & 28.1 & 1.6 & 1.2 to 2.2 \\
\hline Digestive $(150-154,157)$ & 4 & 0 & 4 & 6.5 & 0.6 & 0.2 to 1.6 \\
\hline Liver (155) & 11 & 3 & 8 & 0.3 & 39.7 & 19.8 to 71.0 \\
\hline Respiratory (162) & 17 & 3 & 14 & 7.1 & 2.4 & 1.4 to 3.8 \\
\hline Breast (174) & 1 & - & 1 & 4.0 & 0.3 & 0.01 to 1.4 \\
\hline Reproductive $(179-180,182-183)$ & 3 & - & 3 & 2.3 & 1.3 & 0.3 to 3.9 \\
\hline Urinary (188-189) & 1 & 0 & 1 & 0.3 & 3.7 & 0.1 to 20.8 \\
\hline Haematological (200-208) & 5 & 1 & 4 & 1.6 & 3.2 & 1.0 to 7.4 \\
\hline
\end{tabular}

$\mathrm{CI}$, confidence interval.

these two analyses, and there was no evidence of a significantly increased risk of breast cancer mortality in either.

\section{Discussion}

These results come from a large cohort of PBC patients identified by comprehensive and strict case finding methods. The incidence and prevalence of PBC is high in this part of the UK. A recent study reported an average annual incidence of definite and probable PBC of 43 per million, and an average mid year prevalence of definite and probable PBC of 311 per million in 1987-94 in one of the districts in the study area ${ }^{6}$ : figures of 28.6 and 202.5 per million respectively were found for the whole study area. The subjects were predominantly female, and were diagnosed in their sixth and seventh decades. This is at the upper end of the age range reported elsewhere and may have been affected by the case finding methods used. Many previous studies were based on cases from regional referral centres which would be more likely to include both younger and symptomatic patients, and those with more complex medical histories.

Cancer events were not always reported consistently across sources. However, very few cancer registrations were available after 1993, so a cancer death or hospital diagnosis post-1993 may not have had a matching registration, simply because these data had not yet been released. Nevertheless, the results show that many cancer events were only notified to the cancer registries at the death of the patient. This could be because diagnosis was only made after death, or because cancer registration did not happen after diagnosis as it should. This was investigated by looking at hospital notes, where they were available, to see if their information on cancer diagnosis was similar to registry records. There were some discrepancies between cancer registration and hospital records, but not in a particular direction. There were eight cancer events after PBC diagnosis which were only recorded in hospital notes. However, only five were pre-1993, when registration information was available. Hospital notes will not always contain details of any cancer diagnosis, if care is being entirely dealt with by a general practitioner. These results do not suggest that there was a large problem of non-registration of cancers. There seems little reason to suggest that these problems should be any worse for PBC patients than any other member of the population, so the standardised rates should not be biased.

Cancer events in which cancer registration occurred before the diagnosis of PBC were excluded from the analysis, as the person years 
of experience were only counted after the diagnosis of PBC. However, even if the diagnosis of PBC could have occurred before the cancer was recognised for some of them (making them eligible to be included in the main analysis), the small numbers at any one site would not have made a material difference to the results.

Early work by the group in west of Scotland noted an excess of extrahepatic cancer in patients with $\mathrm{PBC}^{3}$ : the standardised incidence ratio in females was 3.5. Recent studies in Sweden and Denmark found a small but significant excess incidence of all cancers (SIR $=1.6$ and 1.4 respectively), when they excluded cancers diagnosed in the year after PBC diagnosis. ${ }^{79}$ However, a Dutch group found no excess deaths from cancers at all sites $(\mathrm{SMR}=0.7)$ using death certificates as the source of subjects. ${ }^{10}$ In our study there was a small, but statistically significant excess of cancer incidence at all sites in PBC patients, if followed from the time of PBC diagnosis $(\mathrm{SIR}=1.7 ; 95 \%$ confidence interval $(\mathrm{CI}) 1.3$ to 2.2): after excluding the first year of follow up after PBC diagnosis, this SIR was reduced to a non-significant excess. Small but significant excesses were found for cancer mortality at all sites. While the precise size of any excess of all cause cancer mortality or incidence is unclear, the width of the confidence interval suggests that the data from this and other studies are consistent with a small excess cancer risk for PBC patients. Longer term follow up of this cohort may go some way to answer this question.

Some previous studies of hepatocellular cancer (HCC) in PBC using crude incidence rates concluded that the risk of HCC was not particularly high in PBC patients (although high in other forms of cirrhosis), ${ }^{11}{ }^{12}$ while others concluded that the rate of HCC in PBC patients was high and similar to that seen in other females with cirrhosis from other causes. ${ }^{13-15}$ A local study recently noted 16 HCCs arising in $667 \mathrm{PBC}$ patients with a total follow up of 2010 person years. ${ }^{16}$ However, this and the current study are not fully comparable, because although the case definition of PBC is the same, the series of 667 patients was taken from the whole tertiary referral practice of the one liver clinic over 20 years, and the cancer events were found by searching hospital notes as opposed to formal registration.

A few studies have attempted to compare HCC risks via adjusted rates. The Dutch study, based on death registrations, reported a significant excess of HCC mortality SMR $=(25.5) .{ }^{10}$ The large Swedish study found no excess of HCC in terms of incidence (SIR $=2.9$ ) when looking at cancers at least one year after diagnosis of PBC. ${ }^{7}$ However, the Danish study gave significantly increased rates of HCC (SIR $=47) .{ }^{9}$ We found an SIR of 74.3 (95\% CI 32 to 146) and an SIR of 72.7 (95\% CI 29 to 150) when excluding the first year of follow up after PBC diagnosis. The $95 \%$ confidence intervals were based on few events, so we were unable to estimate the excess risk precisely, but it seems likely that there is a large excess risk of liver cancers in PBC patients. Similar results were obtained in this study when looking at cancer mortality rather than incidence rates. The studies which had used adjusted rates found large excesses, except for the Swedish study. It has been suggested that increased risks of HCC are linked to fully developed cirrhosis. ${ }^{76}$ The nine subjects in this study who developed HCC had stage 4 disease: the lack of apparent increase in HCC in the Swedish study may be due to the fact that this study contained many early stage PBC patients.

An American study first reported an excess of breast cancer incidence in PBC patients $(\mathrm{SIR}=4.4)^{5}$; the Scottish group who first reported an excess in all cancers also found excess breast cancer in a larger group of PBC patients (SIR $=3.8) .{ }^{4}$ A link between breast cancer and PBC by the potential role of hormonal factors and immunohistochemical similarities has been suggested, but little evidence has been gathered. ${ }^{17-19}$ However, these early findings were not supported by the results of studies in Italy, ${ }^{13}$ Holland $($ SMR $=0.1),{ }^{10}$ Sweden $($ SIR $=0.9),{ }^{7}$ or Denmark $(\operatorname{SIR}=$ 0.9). ${ }^{9}$ Our study also found little evidence for an excess incidence or mortality from breast cancer, whether or not the first year of follow up after PBC diagnosis was excluded (SIR = $1.1 ; 95 \%$ CI 0.4 to 2.4 for the former).

We found some small excess cancer mortality at sites not previously noted in PBC patients: there was some evidence of additional deaths from respiratory cancer, and lymphomas and leukaemias. Some studies have previously found evidence linking lymphomas to other autoimmune conditions, ${ }^{20}{ }^{21}$ but not to PBC. However, as our results are based on few cancer events, the evidence is not conclusive, and may, indeed, be the result of the multiple comparisons within the analysis. It would nevertheless be interesting to see whether these results are replicated in future studies.

We have considered the risks of cancer at particular sites in four ways: incidence and mortality, and with or without the first year of follow up. The estimates of extra risk were similar whichever method was used. Those based on more years of follow up had more precise estimates, as would be expected. There was a tendency for the estimates based on incidence to be slightly higher than those for mortality, possibly because some of those diagnosed with cancer did not die of it, but the difference was not large.

In conclusion, when considering those cancers whose links with PBC have been studied on a number of occasions, the larger and more rigorous studies concur that it is unlikely that there is a clinically important excess incidence or mortality from any cancer associated with PBC (and specifically breast cancer), with the exception of liver cancer.

Dr JV Metcalf was supported by a Wellcome Trust training fellowship.

\footnotetext{
1 Moreno-Otero R, Lisker-Melman M, Jones AE. Primary biliary cirrhosis. Med Clin North Am 1989;73:911-29. 2 Kaplan MM. Primary biliary cirrhosis. N Engl f Med 1996;
} 
3 Mills PR, Boyle P, Quigley EMM, et al. Primary biliary cirrhosis: an increased incidence of extrahepatic malignancies? F Clin Pathol 1982;35:541-3.

4 Goudie BM, Burt AD, Boyle P, et al. Breast cancer in women with primary biliary cirrhosis. BMF 1985;291 $1597-8$

5 Wolke AM, Schaffner F, Kapelman B, et al. Malignancy in primary biliary cirrhosis: high incidence of breast cancer in affected women. Am F Med 1984;76:1075-8.

6 Metcalf JV, Bhopal RS, Gray J, et al. Incidence and prevalence of primary biliary cirrhosis in the city of Newcastle upon Tyne. Intern f Epidemiol 1997;26:830-6.

7 Loof L, Olov-Adami H, Sparen P, et al. Cancer risk in primary biliary cirrhosis: a population-based study from Sweden. Hepatology 1994;20:101-4.

8 Cuzick J, Babiker AG. Pancreatic cancer, alcohol, diabetes mellitus and gallbladder disease. Int $\mathcal{f}$ Cancer 1989;245: mellitus

9 Sorenson H, Frijs S, Olsen J, et al. Risk of liver and other types of cancer in patients with cirrhosis: a nationwide types of cancer in patients with cirrhosis: a nation
cohort study in Denmark. Hepatology 1998;28:921-5.

10 Van Dam GM, Gips CH. Primary biliary cirrhosis in The Netherlands. Scand f Gastroenterol 1997;32:77-83.

11 Krasner N, Johnson PJ, Portmann B, et al. Hepatocellular carcinoma in primary cirrhosis: report of four cases. Gut 1979;20:255-8.
12 Turissini SB, Kaplan MM. Hepatocellular carcinoma in primary biliary cirrhosis. Am $\mathcal{F}$ Gastroenterol 1997;92:676-8.

13 Floreani A, Biagini M, Chiaramonte M, et al. Incidence of hepatic and extra-hepatic malignancies in primary biliary cirrhosis (PBC). Ital f Gastroenterol 1993,25:4

14 Melia WM, Johnson PJ, Neuberger J, et al. Hepatocellular carcinoma in primary biliary cirrhosis: detection by alphafetoprotein estimation. Gastroenterology 1984;87:660-3.

15 Caballeria L, Pares A, Castells A, et al. Risk of hepatocellular carcinoma in patients with primary biliary cirrhosis. $\mathcal{f}$ Hepatology 1997;26(suppl 1):140

16 Jones D, Metcalf J, Collier J, et al. Hepatocellular carcinoma in primary biliary cirrhosis and its impact on outcomes. Hepatology 1997;26:1138-42.

17 Wilkinson M, Iqbal M, Williams R. Letter. BMF 1986;292: 341.

18 Bergasa N. Primary biliary cirrhosis in patients with breast cancer: studying the link. Med Hypotheses 1998;50:465-72.

19 Sorenson $\mathrm{H}$, Friis $\mathrm{S}$, Olsen J, et al. Risk of breast cancer in men with liver cirrhosis. Am f Gastroenterol 1998;93:231-3.

20 Laakso M, Mutru O, Isomaki H, et al. Cancer mortality in Laakso M, Mutru O, Isomaki $\mathrm{H}$, et al. Cancer mortality in
patients with rheumatoid arthritis. $f$ Rheumatol 1986;13: patients $385-417$.

21 McCurley T, Collins R, Ball E, et al. Nodal and extranodal lymphoproliferative disorders in Sjogren's syndrome. Hum Pathol 1990;21:482-92. 